\title{
The Swift X-ray Cluster Survey
}

\author{
Alberto Moretti* \\ INAF Osservatorio Brera, via Brera 28 20121, Milano \\ E-mail: alberto.moretti@brera.inaf.it \\ Teng Liu, Jun-Xian Wang \\ CAS Key Laboratory for Research in Galaxies and Cosmology, Department of Astronomy
}

Paolo Tozzi, Elena Tundo

INAF Osservatorio di Arcetri

Sergio Campana, Gianpiero Tagliaferri

INAF Osservatorio di Brera

Mauro Giavalisco

Department of Astronomy, University of Massachusetts

\begin{abstract}
The Swift X-ray Cluster Survey (SWXCS) is a catalog obtained using archival data from the Xray telescope (XRT) on board the Swift satellite acquired from 2005 February to 2012 November. We use 3000 observations, covering a total solid angle of $400 \mathrm{deg}^{2}$. We identify extended source candidates in the soft-band $(0.5-2 \mathrm{keV})$ images of these fields using the software EXSdetect, which is specifically calibrated for the XRT data. Extensive simulations are used to evaluate contamination and completeness as a function of the source signal, allowing us to minimize the number of spurious detections and to robustly assess the selection function. The catalog provides positions, soft fluxes, and, when possible, optical counterparts for a flux-limited sample of 263 X-ray group and cluster candidates down to a flux limit of $7 \times 10^{-15} \mathrm{erg} \mathrm{cm}^{-2} \mathrm{~s}^{-1}$ in the soft band. Among them 126 are new detections. Currently, we have collected redshift information for 158 sources ( $60 \%$ of the entire sample). Once the optical follow-up and the X-ray spectral analysis of the sources are complete, the SWXCS will provide a large and well-defined catalog of groups and clusters of galaxies to perform statistical studies
\end{abstract}

Swift: 10 Years of Discovery,

2-5 December 2014

La Sapienza University, Rome, Italy

* Speaker. 


\section{Introduction}

Groups and clusters of galaxies are the most massive, gravitationally bound structures in the universe and their hot intra-cluster medium (ICM) makes them appear as prominent extended sources in the X-ray sky. Therefore, X-ray cluster surveys are among the most efficient tools for constraining cosmological parameters and primordial density fluctuations. A large and complete catalog of groups and galaxy clusters spanning a wide range of redshifts would be crucial to make significant steps toward the understanding of cosmic structure formation and evolution, the chemical and thermodynamical cosmic history of the ICM, and to provide an accurate measurement of cosmological parameters [1] [2] [3]. However, this task is not within the scope of current $\mathrm{X}$-ray missions. None of the major X-ray facilities existing today were designed for surveys, and they have a low efficiency when discovering rare objects like galaxy clusters, particularly at high redshifts. The main characteristics required for an effective X-ray survey mission for extended sources are the following: large field of view (FOV; of the order of $1 \mathrm{deg}^{2}$ ), high angular resolution (of the order of a few arcseconds), low background, and a large effective area (of the order of $10,000 \mathrm{~cm}^{2}$ ). Looking at the near future, the upcoming mission eROSITA [4] will finally provide an X-ray all sky coverage 20 years after the ROSAT All Sky Survey, down to limiting fluxes more than one order of magnitude deeper than ROSAT for extended sources. Therefore eROSITA will considerably increase the number of X-ray groups and clusters particularly at low and moderate redshifts. However, its limiting flux is predicted to be $3.4 \times 10^{-14} \mathrm{erg} \mathrm{cm}^{-2} \mathrm{~s}^{-1}$ after four years of operation, well above the level at which the majority of the high-z clusters, and medium- and high- $\mathrm{z}$ groups, are currently found. In addition, its low effective area above $2 \mathrm{keV}$ severely limits the characterization of the ICM in high-temperature clusters. Currently, the best resource for building X-ray cluster samples is provided by the still increasing archives of the major X-ray facilities Chandra and XMM-Newton. For a review of the ongoing X-ray cluster surveys, up to the year 2012, see Table 1 in [5].

In this framework, we recently presented the Swift X-ray Cluster Survey [5][6] [7]. This is based on the archival data of the X-ray telescope (XRT) on board the Swift satellite. Despite its small collecting area (about one-fifth of Chandra), XRT has two characteristics which make it an efficient instrument for the detection and characterization of extended sources: a low background [8] and a constant angular resolution (with a half energy width of HEW $=18$ ") across the entire FOV [9]. We note that the XRT angular resolution is as good as the resolution of XMM-Newton close to the aim-point, and therefore better than XMM-Newton when averaged over the FOV. To detect and study galaxy clusters, we developed software designed for the detection and photometry of extended sources and optimized for the characteristics of XRT data (EXSdetect, [10]). The source detection method used in EXSdetect is a combination of Voronoi Tessellation and friendof-friend algorithms. This method does not require a priori assumptions about the shape and size of the sources, and it is particularly efficient when applied to X-ray images characterized by many empty pixels. The efficiency of our detection algorithm as a function of the exposure time and the source flux together with the accuracy in the source photometry, are investigated by extensive simulations. Our simulations allow us to evaluate the contamination (number of spurious extended sources) and the completeness of the catalog as a function of the source flux. Moreover we can identify an optimal threshold in the source photometry above which our catalog reaches the re- 


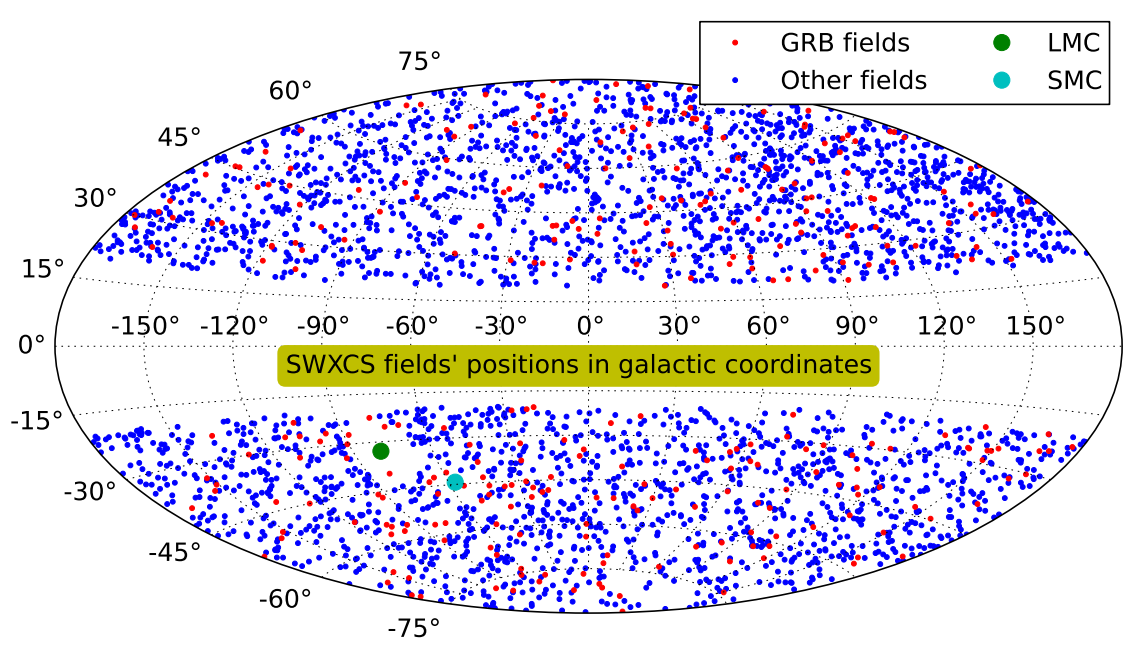

Figure 1: Positions of the 3000 fields of the survey.

quired completeness and purity. This threshold directly provides a position-dependent flux limit for each field, and hence a selection function depending on the source flux for the entire survey. This step is particularly relevant since, once the selection function and the contamination level are accurately predicted, the sample can be used for statistical studies.

\section{The survey}

From the entire Swift XRT archive in the period 2005 February-2012 November, we select all the fields that can be used to build an unbiased, serendipitous X-ray cluster catalog. First, we exclude all of the fields whose Galactic latitude $|\mathrm{b}|<20^{\circ}$. Although these fields could in principle be used to search for bright extended sources, they are typically very crowded, which would cause severe blending problems when the spatial resolution is limited. Moreover, a significant fraction of the soft band emission from groups and clusters would be absorbed by the high HI Galactic column density. The search for the brightest groups and clusters in the Galactic fields will be performed with a different technique in a dedicated paper (A. Moretti et al., in preparation). Second, we exclude the shallow fields whose exposure time is $>3000 \mathrm{~s}$. This limit guarantees 100 background photons in the soft band in each field. This threshold represents the minimum number of photons to sample the background in an X-ray image, which is a critical step to identify extended sources as enhancements of the photon density with respect to the background level. We set a lower limit of 100 to the number of total photons based on extensive simulations of background only images, where we tested the capability of recovering the true background in our algorithm (see [10]). This forces us to discard a large number of fields (see lines in Figure 1) which would have contributed only at very high fluxes $\left(10^{-12} \mathrm{erg} \mathrm{cm}^{-2} \mathrm{~s}^{-1}\right)$ where the expected number of clusters is very low.

A further, fundamental step is to filter out all of the fields that are directly targeting groups or clusters of galaxies, since they would clearly introduce a positive bias toward the detection of extended sources. In principle, one can simply excise the targeted group or cluster and use the rest 

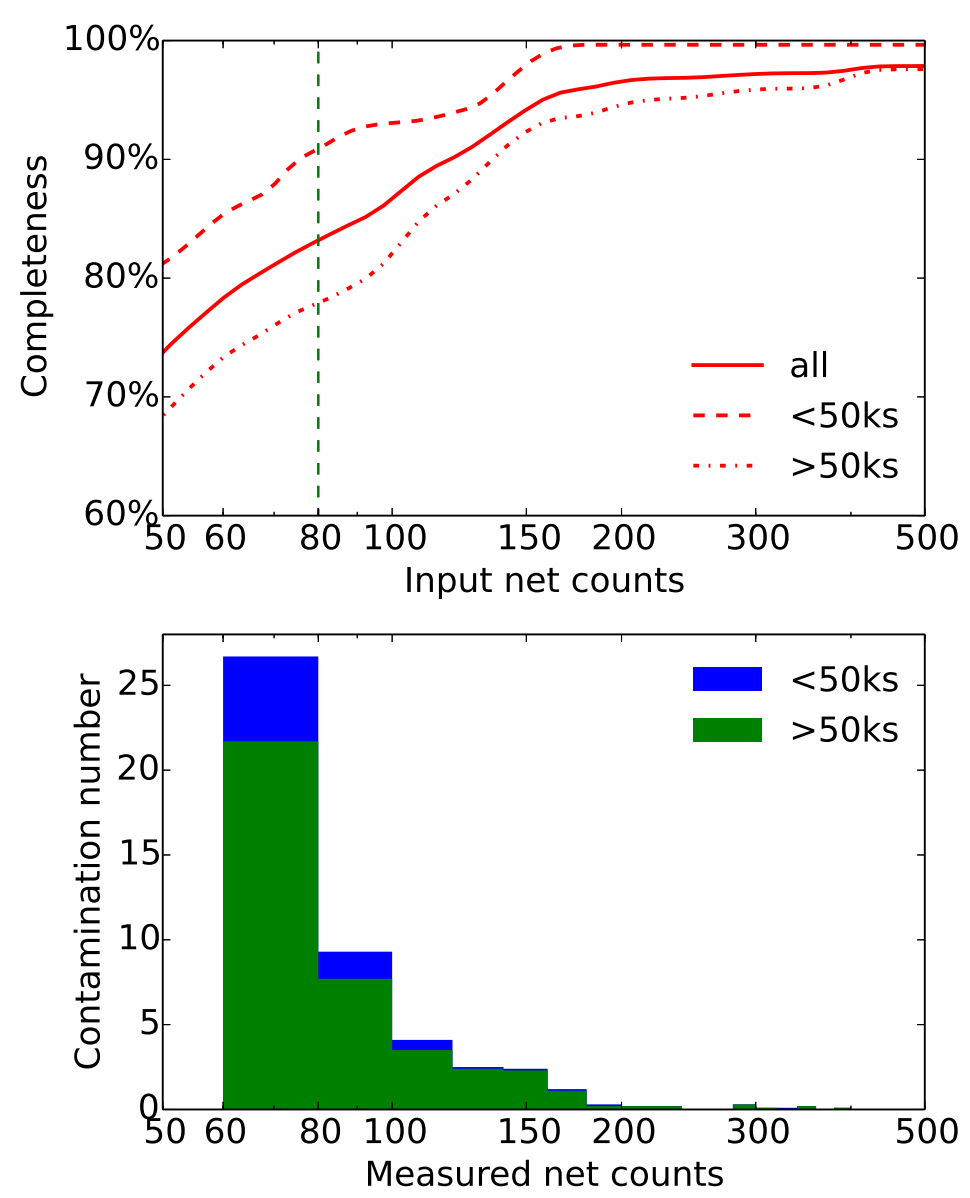

Figure 2: Completeness and purity of the sample

of the field. However, due to the correlation function of dark matter halos, to have an unbiased sample one should also exclude extended sources with similar redshifts. Since this information is not available for many of our sources, we decided to remove all of the fields which, on the basis of the target name and coordinates, are aimed at groups and clusters of galaxies. We also filter out observations targeting nearby galaxies because such galaxies appear as bright extended X-ray sources, whose emission is not associated with ICM but mostly with X-ray binaries and massive star formation events. On the other hand, all of the fields targeting GRBs and active galactic nuclei (AGNs) are included in this survey. We conservatively discard a large number of fields (more than 700) targeting sources whose classification is uncertain. This enables us to avoid any field selection bias, which is a critical requirement for statistical studies and cosmological tests. Our final list consists of 3004 fields that provide a truly serendipitous sampling of the extragalactic sky. The positions in the sky of the aim points of the selected fields are shown in Figure 1.

\section{The catalog}

The XRT data reduction is described in [5]. We consider only the soft-band (0.5-2keV) images 


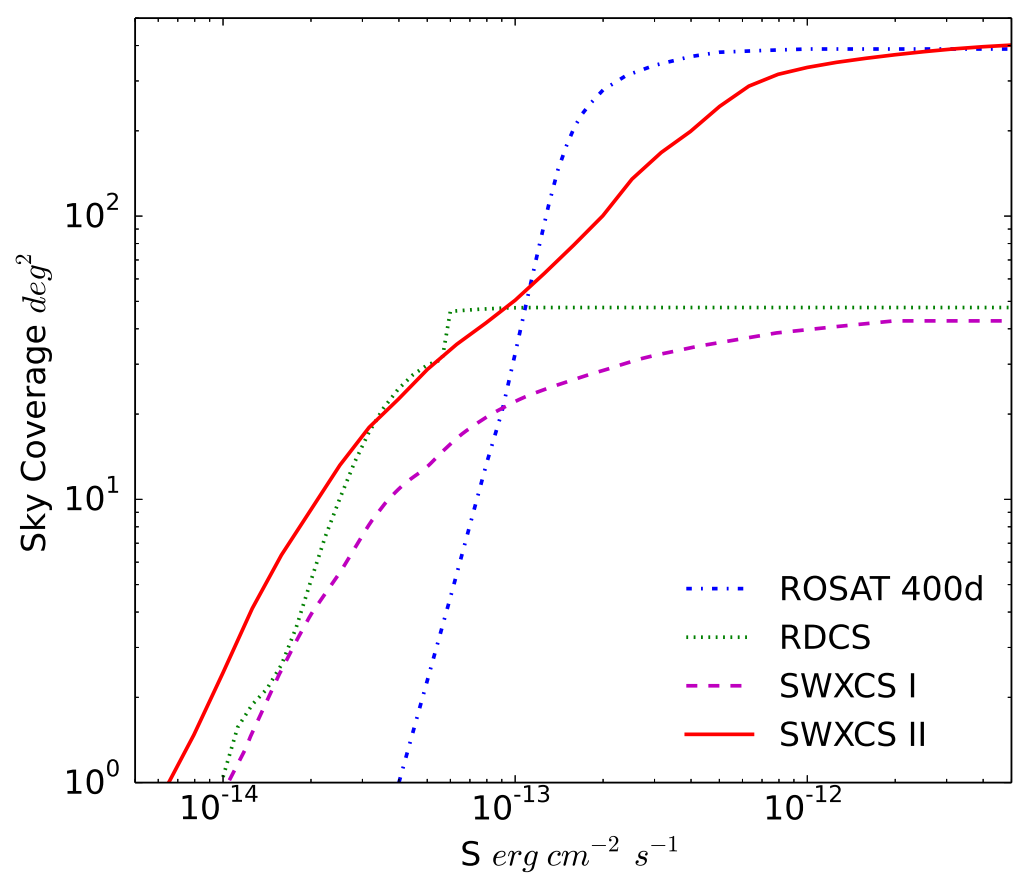

Figure 3: Sky coverage of the survey, compared to other similar works from the ROSAT archive.

for source detection, since the inclusion of the Swift-XRT hard-band images is not useful to identify or to characterize the detected sources.

To detect extended sources we use, for the first time, the EXSdetect software. This algorithm and its performance on the Swift-XRT data are described in full details in [10]. ${ }^{1}$

We achieve strong control of the purity and completeness of the sample thanks to extensive simulations. Simulations are run on a set of synthetic images with the same exposure time distribution and the same background of the selected SWXCS fields. Another important aspect is that the input flux distribution of the simulated sources is taken from real, deep data. Point sources are randomly extracted from a distribution modeled on the number counts found in deep Chandra fields and simulated down to a flux about one order of magnitudes lower than the expected detection limit of the SWXCS[11][12]. The flux distribution of the input extended sources was taken from the number counts of groups and clusters measured in the ROSAT deep cluster survey[13]. Finally, to take into account the different morphologies of extended sources, we modeled the surface brightness of our simulated sources on real images of 10 bright groups and clusters of galaxies observed with Chandra, covering a wide range in ICM temperature (for details see [10]).

In Figure 2, we show the expected completeness (fraction of extended sources recovered at a given value of input net counts), and the number of spurious extended sources expected in the entire SWXCS in bins of net detected counts, in the upper and lower panels, respectively. We also plot the completeness and contamination separately for fields with exposure times above and below

\footnotetext{
${ }^{1}$ The software is publicly available and currently updated on the SWXCS Web site (http://www.arcetri.astro.it/SWXCS/ and http://swxcs.ustc.edu.cn).
} 
$50 \mathrm{ks}$, which account for $10 \%$ and $90 \%$ of all the fields, respectively. We note that most of the incompleteness and most of the spurious sources come from the $10 \%$ deepest fields. The reason for this is the less efficient performance of EXSdetect in the presence of high background and crowded fields. For the entire SWXCS, completeness falls below $90 \%$ at about 130 net counts, and reaches $83 \%$ at 80 net counts. Meanwhile, the contamination number increases rapidly below 80 net counts. Above 80 net counts, the total number of spurious extended sources in the entire survey at any flux is estimated to be about 20, most of which have less than 150 net counts. Therefore, although the completeness is still high and robustly measured below 80 counts, we conservatively set the detection threshold of our catalog to 80 net counts within the source region as defined by the EXSdetect algorithm to keep a low number of spurious detections.

For each field, the minimum detectable count rate, computed as 80/texp, corresponds to a position-dependent flux limit obtained by multiplying this number by the field energy conversion factor (ECF) at the aimpoint in the $0.5-2 \mathrm{keV}$ band (which accounts for Galactic absorption and is computed for an average thermal model with a temperature of $5 \mathrm{keV}$, a metal abundance of $\mathrm{Z}=0.3$ , and a redshift $\mathrm{z}=0.4$ ), and by the normalized exposure map, which accounts for the vignetting effects. Since the ECFs weakly depend on the spectral parameters a flux-limit map can be obtained for each field. The sum of the flux-limit maps of the entire set of fields considered in SWXCS provides the sky coverage of the survey as a function of the energy flux. The sky coverage of SWXCS is shown in Figure 3 (solid line). We define the flux limit of the survey $S_{\text {lim }}$ as the flux at which the sky coverage falls below $1 \mathrm{deg}^{2}$. This corresponds to a flux of $S_{\text {lim }}=7 \times 10^{-15} \mathrm{erg}$ $\mathrm{cm}^{-2} \mathrm{~s}^{-1}$, The maximum solid angle covered by the survey is $\sim 400 \mathrm{deg}^{2}$, reached above a flux of $\mathrm{S}>3 \times 10^{-12} \mathrm{erg} \mathrm{cm}^{-2} \mathrm{~s}^{-1}$

If we compare the sky coverage of the SWXCS with previous deep X-ray surveys of galaxy clusters, we find that the SWXCS reaches a depth similar to the ROSAT Deep Cluster Survey [13] and a width similar to the ROSAT 400d survey [14], as shown in Figure 3 (dotted and dot dashed lines).

By running EXSdetect (version 3.0) on the soft-band images of the 3000 fields, we obtain 430 extended source candidates with a soft-band photometry larger than 80 net counts in the source region defined by the EXSdetect algorithm itself. According to the expected performance of EXSdetect, the directly obtained source catalog should have only about 20 spurious sources. Apart from this budget, however, we know that we have spurious sources associated with circumstances not included in the simulations, but which can be easily identified and filtered out. Another effect that is not considered in the simulations, for obvious reasons, is associated with the wings of bright clusters which only partially fall in the FOV of the XRT The first class of these sources is the piled-up targets, which we can always safely assume to be bright AGNs or GRBs. Nearby $(\mathrm{z}<$ $0.05)$ galaxies constitute another source of contamination for our sample. Another kind of spurious detection is caused by bright optical sources (generally stars) whose intense optical/UV emission induces significant spurious charge load in the CCD. Such sources are automatically screened by the Swift-XRT pipeline. However, this process often leaves a ring-like signature that is classified as extended by our algorithm but can be immediately spotted by visual inspection. At the end of this cleaning procedure, we have removed 167 sources due to effects that could not be included in the simulations. In principle, these effects could be implemented in the reduction pipeline with some additional effort. However, in our case, due to the limited number of sources in the SWXCS, 
a manual check a posteriori is feasible and the automatization of this filtering process is not crucial at this stage of the project. After this step is completed, we are left with 263 group and cluster candidates whose properties are well described by the completeness and contamination function obtained with our simulations.

\section{Collecting cluster redshifts}

Overall, about half (137) of the 263 SWXCS sources were previously identified as groups or clusters of galaxies, while 126 SWXCS sources are new cluster and group candidates. Thanks to these identifications, we are able to recover the redshift information for a significant fraction of our sample. We collect spectroscopic or photometric redshifts for 130 of our sources. Moreover, to increase the number of available redshifts, we also search in NED catalogs for single galaxies with published redshifts not associated with previously known clusters within a search radius of 7' from the X-ray centroid of our sources. We find 50 galaxies with measured redshifts for 47 of our sources as a complement to the redshifts obtained from cluster counterparts. In 35 cases where we have both cluster and galaxy counterparts, the galaxy redshifts are consistent with those of clusters. In the 12 cases where no cluster counterpart is found, we tentatively assign the galaxy redshift to our X-ray source. If we also consider the X-ray redshift derived from the Telescopio Nazionale Galileo (TNG) observations and the X-ray spectral analysis of the sources [6], then we have a total of 158 sources with redshifts, from optical spectroscopy or photometry or from Xray spectral analysis. Therefore, about $60 \%$ of our sample has redshift information. The highest redshift measured by optical spectroscopy is $z=0.92$ for XCS J142908.4+424128.9 [15]. This confirms that the depth of our catalog is sufficient to select clusters up to $\mathrm{z} \sim 1$. Another high redshift source candidate is SWXCSJ011432-4828.4, whose redshift is measured to be $0.97 \pm$ 0.02 from X-ray spectral analysis. The presence of clusters at $\mathrm{z} \sim 1$ is not unexpected in the SWXCS, given the non negligible sky-coverage of the SWXCS at low fluxes. Based on previous results from the RDCS, we expect of the order of 10 clusters with $z>1$. In addition to the few sources already mentioned, we already identified a sample of high redshift candidates among the sources with SDSS images but no optical counterparts. The next step of our project is to increase the number of identifications and redshift measurements, in order to use our sample for statistical studies and cosmological tests. We have started an extensive follow-up program to obtain sensitive, multiband imaging photometry of the SWXCS sample. Our immediate goal is to measure the integrated properties of the stellar populations of the galaxies through SED fitting. We will also explore the correlation of the galaxy properties with those of the hosting cluster and their evolution with redshift. The planned observations consist of deep CCD images in the UBVRIz filters which, in the redshift range $0.3<\mathrm{z}<1$, probe the restframe spectral range from mid- and far-UV to optical wavelengths. This choice allows us to properly sample the wavelengths across the $4000 \mathrm{~A}$ break, which are key for accurate measurements of the integrated stellar mass, star formation rate, average dust obscuration, and luminosity weighted age through SED fitting. Currently, we have observed a total of 41 groups and clusters, 11 in the south and 30 in the north one. We plan to release the reduced and calibrated images and source catalogs of the first year of observations in early 2015. The program will continue throughout the coming years. We finally note that we can add a significant number of redshifts by extending the X-ray spectral analysis to the entire sample. When 
the optical follow-up and the extension of the X-ray spectral analysis is completed, the SWXCS will provide a large and well defined catalog of groups and clusters of galaxies to perform statistical studies of cluster properties and tests of cosmological models. All of the results of the SWXCS are publicly available on http://www.arcetri.astro.it/SWXCS or http://swxcs.ustc.edu.cn, including machine readable tables and the EXSdetect code.

\section{References}

[1] A. Vikhlinin, A. V. Kravtsov, R. A. Burenin et al. (2009), Chandra Cluster Cosmology Project III: Cosmological Parameter Constraints, ApJ, 692, 1060

[2] A. Mantz, S.W. Allen, D. Rapetti, H. Ebeling, (2010), The observed growth of massive galaxy clusters - I. Statistical methods and cosmological constraints, MNRAS, 406, 1759

[3] S.W. Allen, A.E. Evrard, \& A.B. Mantz, (2011), Cosmological Parameters from Observations of Galaxy Clusters, ARA\&A, 49, 409

[4] P. Predehl, R. Andritschke, H. Bohringer et al. (2010), eROSITA on SRG, Proc. SPIE, 8145

[5] E. Tundo, A. Moretti, P. Tozzi et al. (2012), The Swift X-ray Telescope Cluster Survey: data reduction and cluster catalog for the GRB fields, A\&A, 547, A57

[6] P. Tozzi, A. Moretti, E. Tundo et al. (2014), The Swift X-ray Telescope Cluster Survey. II. X-ray spectral analysis, A\&A, 567, A89

[7] T. Liu, P. Tozzi, E. Tundo et al. (2015), The Swift X-Ray Telescope Cluster Survey. III. Cluster Catalog from 2005-2012 Archival Data, ApJS, 216, 28

[8] A. Moretti, C. Pagani, G. Cusumano, et al. (2009), A new measurement of the cosmic X-ray background, $A \& A, 493,501$

[9] A. Moretti, M. Perri, M. Capalbi et al. (2007), The swift-XRT imaging performances and serendipitous survey, Proc. SPIE, 6688

[10] T. Liu, P. Tozzi, E. Tundo, et al. (2013), EXSdetect: an end-to-end software for extended source detection in X-ray images: application to Swift-XRT data, A\&A, 549, A143

[11] A. Moretti, S. Campana, D. Lazzati, G. Tagliaferri, (2003), The Resolved Fraction of the Cosmic $X$-Ray Background, ApJ, 588, 696

[12] B.D. Lehmer, Y.Q. Xue, W.N. Brandt et al. (2012), The 4 Ms Chandra Deep Field-South Number Counts Apportioned by Source Class: Pervasive Active Galactic Nuclei and the Ascent of Normal Galaxies, ApJ, 752, 46

[13] P. Rosati, R. della Ceca, C. Norman, R. Giacconi (1998), The ROSAT Deep Cluster Survey: The $X$-Ray Luminosity Function out to $z=0.8$, ApJL, 492, L21

[14] R.A. Burenin, A. Vikhlinin, A. Hornstrup et al. (2007), The 400 Square Degree ROSAT PSPC Galaxy Cluster Survey: Catalog and Statistical Calibration, ApJS, 172, 561

[15] N. Mehrtens, A.K Romer, M. Hilton et al. (2012), The XMM Cluster Survey: optical analysis methodology and the first data release, MNRAS, 423, 1024 